\title{
Activités
}

8-1 | avril 2011

Varia

\section{Pénibilités des activités de service et santé des Agents Spécialisés des Écoles Maternelles}

Des évolutions avec l'âge

Infant School Assistants : the strenuous nature of their service activities, their health and how these change with age

Catherine Delgoulet, Annie Weill-Fassina et Céline Mardon

\section{OpenEdition}

Journals

Édition électronique

URL : http://journals.openedition.org/activites/2493

DOI : $10.4000 /$ activites. 2493

ISSN : $1765-2723$

Éditeur

ARPACT - Association Recherches et Pratiques sur les ACTivités

Référence électronique

Catherine Delgoulet, Annie Weill-Fassina et Céline Mardon, «Pénibilités des activités de service et santé des Agents Spécialisés des Écoles Maternelles », Activités [En ligne], 8-1 | avril 2011, mis en ligne le 15 avril 2011, consulté le 20 avril 2019. URL : http://journals.openedition.org/activites/2493 ; DOI : 10.4000/activites.2493

\section{(c) (i) (2)}

Activités est mis à disposition selon les termes de la licence Creative Commons Attribution - Pas d'Utilisation Commerciale - Pas de Modification 4.0 International. 


\title{
Pénibilités des activités de service et santé des Agents Spécialisés des Écoles Maternelles. Des évolutions avec l'âge
}

\author{
Catherine Delgoulet \\ Laboratoire Adaptations Travail-Individus \\ Université Paris Descartes, 71 avenue Edouard Vaillant, 92100 Boulogne-Billancourt \\ catherine.delgoulet@parisdescartes.fr \\ Annie Weill-Fassina \\ EPHE et CREAPT \\ awl@noos.fr

\section{Céline Mardon} \\ CREAPT - CÉE, \\ Immeuble «Le Descartes I », 29 promenade Michel Simon, 93166 Noisy-Le-Grand Cedex \\ celine.mardon@cee-enpc.fr
}

\begin{abstract}
Infant School Assistants: the strenuous nature of their service activities, their health and how these change with age. The study examined the working conditions of Infant School Assistants (ISAs) who perform three types of task : cleaning, canteen work and educational classroom assistance in schools. It was carried out through a local authority and the aim was to understand the different types of strain resulting from ISAs' strenuous working conditions and how these change with age. These results were to be used to predict the consequences of ageing and to devise preventive actions to avoid the onset of disease and accidents in the workplace. The central hypothesis was that the strenuous nature of their activities is linked to the combination and diversity of tasks. This hypothesis was based on previous research carried out on the concept of "strenuous working conditions" for similar jobs and activities (such as cleaning, or institutional catering). Data on different work activities was obtained through interviews, observation and questionnaires. The results show that despite the real interest the ISAs take in their job, they are weighed down by the multiplicity of tasks and managers. These factors both contribute to increased difficulties at work by creating task overlaps or interference, and by cutting the ISAs off from the "educational community". Hence, the results highlight the impact on perceived health and safety at work, especially as ISAs age.
\end{abstract}

KEYWORDS

Work organization, service activities, workload, health, age, Infant School Assistants

La recherche-action dont il sera rendu partiellement compte ici se déroule en 2008-2009 dans le cadre d'une intervention auprès d'une mairie d'une commune d'Ile de France. Celle-ci a commandité une réflexion sur les conditions de travail des Agents Spécialisés des Ecoles Maternelles (ASEM); le but étant d'anticiper les conséquences du vieillissement de cette population et réfléchir à des pistes d'action pour prévenir la survenue de maladies professionnelles et accidents du travail. L'objectif poursuivi dans cet article est de montrer les pénibilités du travail de restauration, entretien et éducation réalisé par les ASEM en s'attachant plus particulièrement à leur évolution avec l'âge et leurs 
conséquences sur la santé.

\section{1.- De la pénibilité aux pénibilités du travail}

La notion de "pénibilité » devient très prégnante dans le monde du travail et les négociations sociales au début des années 2000, dans le cadre des réflexions menées en parallèle de la mise en place de la loi de 2003 sur l'allongement de la vie au travail. Les rapports Struillou (2003) puis Lasfargues (2005) définissent en détail les facteurs de la pénibilité du travail pour l'un et leurs conséquences en termes d'atteinte à la santé pour l'autre. Ils retiennent essentiellement des critères « objectifs » de pénibilité, ou considérés comme tels : durée journalière ou période d'exposition à des facteurs de risques que sont le travail de nuit ou en horaires alternants, le travail répétitif sous cadence imposée, les contraintes physiques et posturales, le port de charges lourdes, les outils vibrants, l'exposition au bruit, aux produits toxiques. Ils considèrent les effets de la pénibilité en se focalisant sur les accidents du travail, les maladies professionnelles, et le taux de mortalité. Ces travaux considèrent la pénibilité comme « des expositions professionnelles pesant sur l'espérance de vie sans incapacité oulet la qualité de la vie » (Struillou, 2003). Ils permettent de dresser un panorama large des conséquences de la pénibilité du travail tout au long du parcours professionnel sur la santé des travailleurs à long terme. Le concept de «pénibilité » défini ainsi est un outil pour la réparation et le dédommagement des mauvaises conditions de travail éprouvées au cours de la vie professionnelle. Il a été et est encore un outil précieux dans les négociations sectorielles sur les départs anticipés en retraite pour pénibilité du travail.

Toutefois, la pénibilité telle que définie dans ces travaux ne couvre qu'une partie de ce que peut recouvrir ce concept. En reprenant la définition du mot (Rey \& Rey-Debove, 1989), on est renvoyé à l'adjectif de la même racine «pénible » qui qualifie à la fois: a) «ce qui se fait avec peine, fatigue et difficulté » où la dimension physique est fortement présente; et b) «ce qui cause de la peine, de la douleur ou de l'ennui; qui est difficile moralement » faisant, en miroir, référence à la dimension psychique. Ces deux faces nous semblent d'autant plus intéressantes à appréhender et opérationnaliser lorsque l'on se place non plus dans une démarche de compensation, mais dans une démarche de prévention. Molinié et Volkoff (2003) préfèrent ainsi parler « des pénibilités » en faisant référence à un ensemble de situations de travail qui sont difficiles à vivre durablement. Dans le cadre de leur réflexion sur les liens possibles entre départs précoces en retraite et pénibilités du travail, ils distinguent (Molinié \& Volkoff, 2006) :

- les astreintes potentiellement pathogènes, invalidantes à long terme, présentes à diverses périodes du parcours professionnel ou tout au long, qui renvoient à la définition de la pénibilité présentée juste avant (Struillou, 2003);

- un vécu difficile des dernières années de vie professionnelle, nommé aussi la «pénibilité vécue »;

- des difficultés, en raison d'un état de santé déficient diagnostiqué par un médecin ou relevant de troubles ressentis par le salarié, qui gênent dans le travail.

Ces pénibilités permettent d'appréhender les dimensions « objective » et « subjective » du concept en redonnant du poids à la diversité des perceptions des situations de travail et donc des actions à mettre en œuvre (hormis les départs précoces en retraite).

Dans cet article, nous nous intéresserons aux pénibilités tout au long de la vie professionnelle et étendrons la dimension de « vécu difficile » à l'ensemble du parcours professionnel. Nous entendrons donc par évolutions des pénibilités, aussi bien l'évolution des difficultés ressenties par les personnes dans leur travail que les changements de leur état de santé et les gênes occasionnées ou les modifications des risques encourus. 


\section{2.- Conditions de travail, santé et âge dans les métiers des services}

\section{1.- La fonction d'ASEM : une activité de services pluridimensionnelle}

La fonction des ASEM, que nous avons observées, est composée de trois missions (entretien, restauration et aide éducative auprès des enfants) qui relèvent toutes trois du secteur des services. Dans ce secteur, aujourd'hui, plus de $70 \%$ des salariés sont en contact avec un tiers (Guignon \& HamonCholet, 2003). La caractéristique de ces activités est de mettre les destinataires du service au centre des préoccupations. Elles n'en revêtent pas moins des caractéristiques sociales et fonctionnelles très différentes.

En nous appuyant sur la catégorisation des «activités marchandes et non marchandes » proposée par Gorz (1988) ${ }^{1}$ il apparaît que les trois missions des ASEM ne répondent pas, d'un point de vue sociologique, aux mêmes types de demandes, ne présupposent pas le même type d'activité, ni le même type de relations avec les destinataires.

L'entretien et la restauration, s'il s'agissait de services à des particuliers privés, seraient assimilés à un «travail de serviteur, de type domestique », dans la mesure où les travaux demandés pourraient être exécutés par les demandeurs eux-mêmes. Mais la fonction d'ASEM relève de la sphère ou du service public où les principes d'égalité et d'équité sont centraux et guident les prestations proposées (Weller, 1999). Ces missions sont alors confiées aux Agents au nom de la division du travail, ce qui permet à d'autres acteurs du système éducatif de développer d'autres activités spécialisées dans de bonnes conditions. S'exerçant ici dans un milieu collectif, ces missions impliquent « un savoir-faire professionnel avec un outillage très performant » (Gorz, op.cit, p. 222), et des règles spécifiques qui en font la qualification, "les services dépassant en qualité et en quantité ceux que les gens pourraient se fournir eux-mêmes par une même quantité de leur propre travail » (Gorz, op.cit, p. 221). Il n'en reste pas moins qu'il s'agit, selon Gorz, de travaux qui offrent peu de perspectives de développement tant sur le plan personnel que social; de plus, sans être au rendement, il s'agit de fournir une quantité de travail contractuelle en un temps mesurable, mais aussi de faire éventuellement plaisir aux personnes qui en bénéficient. Le travail se trouvant délégué, il relève d'une activité de service sans relation personnelle dans son déroulement, qui peut s'exercer hors de la présence des destinataires pour l'entretien en tout cas et pour les tâches de préparation de restauration (ce qui contribue à rendre le travail invisible). Néanmoins, les usagers de l'école dans leur ensemble peuvent être considérés comme facteurs des conditions de travail des agents (Falzon \& Lapeyrière, 1998). Par exemple, l'état dans lequel ils laissent les locaux influe sur les activités d'entretien, de même que la manière dont se tiennent les enfants sur le service à la cantine.

La mission éducative répond institutionnellement aux besoins d'aide des enseignants mais les bénéficiaires en sont également les élèves. Ses objectifs prioritaires sont de répondre aux besoins de soins, d'aide et de formation des élèves (à l'entrée, et à la sortie, en classes ou au moment des repas). Ceux-ci sont les objets du travail (Falzon \& Lapeyrière, 1998). Selon Gorz, l'organisation de cette mission se déroule à la demande de l'encadrement scolaire mais il est impossible d'en quantifier l'efficacité. Par rapport aux élèves, «l'ajustement de l'offre et de la demande relève d'une relation de personne à personne et non de l'exécution d'actes prédéfinis et quantifiables. » (p. 229). La relation pédagogique implique l'autonomie car "la manière de s'impliquer dans la prestation ne peut être ni produite à volonté, ni achetée ni apprise ni codifiée » (p. 231). Cette relation peut déborder de la sphère publique par une entente plus personnelle qui n'est pas comme précédemment uniquement de

1. Pour A. Gorz, se situant dans une perspective sociologique et politique, la « rationnalité économique paraît applicable aux activités qui a) créent de la valeur d'usage; b) en vue d'un échange marchand; c) dans la sphère publique; d) en un temps mesurable et avec un rendement aussi élévé que possible ». Il analyse et caractérise en ce sens des activités marchandes et non-marchandes (hors production ouvrière). En particulier, pour ce qui nous intéresse ici d'un point de vue ergonomique, il distingue et schématise le travail de serviteur et les fonctions de services, (de gardien de la paix, du pompier, de soins, d'aide, d'enseignement) quant à leur statut social et leurs rapports aux autres. 
l'ordre du faire plaisir. Aussi, l'intervention d'un tiers pourrait-elle être vécue comme une intrusion.

D'un point de vue ergonomique, on a constaté que dans cette relation entre prestataire, usager et bénéficiaire, pouvait parfois se construire, une situation de coopération définissable selon quatre caractéristiques (Falzon \& Lapeyrière, 1998): un objet de travail commun aux prestataires et bénéficiaires (même si les projets ne sont pas identiques); l'inégalité des moyens (physiques et cognitifs) pour atteindre l'objectif; l'existence de moyens complémentaires de part et d'autre (la relation d'aide instituée socialement exigeant à la fois la disponibilité du spécialiste, la sincérité de la demande du bénéficiaire); et de part et d'autre, le devoir de mise en œuvre des moyens disponibles pour satisfaire la demande. Ces situations peuvent concerner à des échelles diverses tant les élèves que le corps enseignant.

\section{2.- Entre contraintes organisationnelles et pénibilités physiques}

À notre connaissance, peu d'études se sont intéressées à la population des ASEM et à leurs conditions de travail. La première identifiée, de Barbier-Le Déroff $(1998,2007)$, est une ethnographie du travail des ASEM et traite des rapports « temps et activité de travail ». Elle souligne notamment les temporalités multiples auxquelles sont soumises les ASEM : a) des temps de travail fluctuant d'une année à l'autre, d'une école à l'autre, ou d'une période scolaire à une période de petites ou grandes vacances; b) une grande répétitivité des cycles d'activité (le nettoyage des sols est réalisé deux fois par jour) associés à de nombreux imprévus (en fonction de la météo, les enfants se salissent plus ou moins dans la cour de récréation ce qui peut à la fois augmenter le temps passé à nettoyer des sols et à changer les enfants); c) une nécessité absolue de respecter certaines butées temporelles (horaire d'entrée à l'école, récréations, repas, sieste, horaire de sortie...) définies par l'employeur (la mairie), les enseignants ou les usagers (les enfants ou les parents). Ces différents temps occasionnent des difficultés d'anticipation de l'activité future; difficultés considérées par l'auteur comme facteur potentiel d'accroissement de la fatigue et de baisse de la qualité du service rendu (omissions, erreurs, tension avec les enfants).

Plus récemment, Le Floc'h (2006) dresse un bilan de la satisfaction professionnelle des personnels éducatifs (professeurs des écoles, ASEM et animateurs des centres de loisirs) exerçant dans 18 écoles d'une grande ville française. Sur la base d'une enquête par questionnaire, elle montre que si l'ensemble des personnels a une perception positive de leur qualité de vie au travail (quel que soit l'âge) et de leur santé (évaluée ici par un indice de «bien-être subjectif »), les ASEM expriment un niveau de qualité de vie et de bien-être inférieur à celui des enseignants et animateurs. Elle en conclut que «La pratique d'un métier où l'on est un des acteurs de l'éducation des enfants reste encore fortement structurante et positive pour chacun. [...] Les ASEM nécessitent une attention particulière pour ne pas être les oubliées de ce collectif de travail. Leur qualité de vie au travail mériterait d'être analysée en particulier sous les éléments organisationnels » (p. 218).

Dans une situation pour partie similaire, Genest (2009) mène un travail auprès d'agents d'entretien des écoles pour concevoir un programme de formation aux diverses tâches de nettoyage et répondre de ce qu'il nomme un «paradoxe »: pourquoi des tâches considérées par tous comme «simples, transparentes et sans consistance » sont-elles sources de difficultés pour des agents dans leur réalisation? Par une approche issue de la didactique professionnelle, l'auteur montre que le travail des personnels d'entretien est à dominante motrice et relève à la fois de savoirs techniques et de savoirfaire d'organisation, mais surtout de réorganisation des tâches en fonction des perturbations au cours de la journée de travail (activités pédagogiques plus ou moins salissantes, disponibilité variable des salles, météo). Il note par ailleurs, sans l'étudier de manière systématique, qu'au-delà des tâches prescrites d'entretiens viennent se greffer des tâches éducatives informelles et auto prescrites (relatives à l'apprentissage de la propreté aux enfants) qui semblent donner un « supplément d'âme » au travail de ces agents.

Dans ces trois études, les liens entre organisation du travail et santé affleurent en filigrane sans être 
directement traités: Barbier-Le Déroff (1998) et Genest (2009) insistent sur la complexité organisationnelle du travail des ASEM, ou des agents d'entretien des écoles, et leurs probables conséquences en termes de fatigue, d'irritabilité ou de difficulté dans le travail; Le Floc'h (2006) remarque au travers d'une évaluation d'éléments subjectifs de santé le poids de l'organisation sur le sentiment de bien-être. L'ensemble de ces travaux signale également la place particulière accordée aux enfants dans cette activité de service.

Dans le secteur de la restauration collective (où les tâches sont proches de celles effectuées par les ASEM lors de leur mission de restauration), les travaux soulignent la pénibilité psychosociale (sans la définir vraiment, Pehkonen, Takala, Ketola, Viikari-Juntura, Leino-Arjas, Hopsu et al., 2009) et surtout physique du travail en cuisine en liant notamment cette dernière à l'inadéquation du mobilier de préparation des plats (hauteur des tables de découpe, hauteur des fours, etc.; Pekkarinen \& Anttonen, 1988). Ils constatent également que les salariés ont de nombreux problèmes musculo-squelettiques; par exemple: l'étude de Haukka, Leino-Arjas, Solovieva, Ranta, Viikari-Juntara, Riihimaki (2006), réalisée auprès de 263 salariés de cuisines municipales de quatre grandes villes de Finlande, rapporte un taux de déclaration de douleurs musculo-squelettiques durant les 3 mois précédents l'enquête de $87 \%$ de la population totale.

Dans le secteur du nettoyage, la dimension physique du travail est à nouveau mise en avant. Elle est étudiée en lien avec les contraintes biomécaniques qu'elle engendre, par la seule réalisation des tâches (Laursen, Sogaard, \& Sjogaard, 2003), ou par l'utilisation des outils et équipements de nettoyage plus ou moins adéquats (Woods \& Buckle, 2005). Une revue de littérature (Kumar \& Kumar, 2008) reprend les travaux publiés entre 1979 et 2005 dans la base de données Ergonomics Abstracts. Cette synthèse définit le travail de nettoyage comme un travail physique, qui suppose une activité musculaire statique et dynamique, des mouvements répétitifs des bras et des mains, le maintien de postures difficiles (courbures et torsions), des efforts en pression ou port de charge. L'analyse bibliographique permet également d'identifier des facteurs potentiels de survenue de TMS, en proposant un modèle selon lequel la réponse physiologique des tissus musculaires à la sollicitation d'une charge physique dépend d'éléments physiologiques et psychologiques individuels variant en fonction a) du contexte organisationnel et psychosocial; b) des facteurs individuels (âge, santé, anthropométrie, niveau d'éducation...) ; c) des procédures de travail, des environnements physiques de travail, des outils et méthodes.

Ce modèle a le mérite de prendre en compte le caractère multidimensionnel du développement des TMS, et permet d'avancer que d'autres éléments, hormis la dimension biomécanique, induisent des troubles de la santé. Deux problèmes organisationnels ou de gestion des ressources humaines sont ainsi identifiés: la monotonie des tâches et l'intensité du travail liées à l'industrialisation du nettoyage; l'absence ou la grande difficulté à construire une carrière professionnelle dans un métier où les possibilités d'évolution sont rares. Toutefois, ce modèle linéaire considère que l'intervention sur un des facteurs permet d'améliorer la prévention des risques de TMS. Il ne permet pas de comprendre comment se combinent les différents facteurs identifiés, comment ces combinaisons accentuent ou pas les troubles à court ou long terme, et comment l'intervention sur l'un d'entre eux peut avoir des effets positifs ou négatifs sur le système en son entier (Daniellou, 2010).

Dans les deux secteurs, une caractéristique semble commune : la population de salariés est essentiellement constituée de femmes, plutôt âgées, peu ou pas qualifiées. Ceci en fait un élément particulier à prendre en compte :

- puisqu'aux pénibilités physiques et aux contraintes organisationnelles s'ajoute le processus de vieillissement «normal » de cette population, faisant d'elle un groupe aux risques de TMS accrus (Ilmarinen, 1994);

- parce que le travail dans ce secteur est souvent considéré comme une extension de leur activité domestique (Puech, 2007) avec les conséquences identifiées depuis longtemps en termes de nonreconnaissance de compétences professionnelles spécifiques ou de maladies professionnelles 
(Guilbert, 1966; Messing, Chatigny, \& Courville, 1998).

Cette dimension n'est pas traitée dans les travaux réalisés auprès des ASEM, précédemment cités, mais nous pouvons faire l'hypothèse que certaines similitudes pourront être retrouvées.

Comme nous l'avons déjà dit, la fonction d'ASEM associe différentes missions qui correspondent à différentes modalités de relations de service (en présence ou non des bénéficiaires). Des conditions de travail difficiles d'un côté, et notamment les difficultés de gestion temporelle des tâches compte tenu des imprévus mais aussi des butées temporelles strictes, et les fragilités de santé de l'autre, ont été mises en évidence sans que des liens entre ces deux dimensions ne soient faits. À l'inverse, des études plus ciblées sur la tâche de restauration collective ou d'entretien ont souligné l'effet délétère de ces tâches sur la santé des salariés (survenue de TMS), sans pouvoir traiter l'effet du cumul des missions tel qu'il existe dans le travail des ASEM. Dans la plupart des cas, les évolutions avec l'avancée en âge ne sont pas ou peu considérées.

Dans ces conditions, l'objectif de cet article est d'étudier les liens potentiels entre conditions de travail, santé et âge en situation de cumul de missions. Notre hypothèse est que le cumul d'astreintes physiques et psychologiques lié aux interférences entre missions des ASEM pourrait accroître les pénibilités du travail (douleurs, gênes dans le travail, insatisfaction, maladies professionnelles, accidents) et être source de risques pour leur santé et leur sécurité, d'autant plus importants avec l'avancée en âge.

\section{3.- Positionnement du problème à la mairie de $\mathrm{V}$.}

\section{1.- Des symptômes « incompréhensibles » de pénibilités à plus ou moins long terme}

S'appuyant sur les chiffres du Bilan Social de l'année précédant l'étude, la mairie part de plusieurs constats pour formuler sa demande:

- environ $60 \%$ des agents exerçant des fonctions d'ASEM dans les services municipaux ont plus de 40 ans (sur plus de 150 agents);

- ces agents ont cumulé sur l'année, 1026 jours d'arrêt pour raison de santé (essentiellement des TMS) ou pour accident de travail;

- neuf d'entre eux ont déclaré une maladie professionnelle et 6 sont en attente d'un reclassement professionnel pour raisons médicales, après avoir été reconnus inaptes à leurs fonctions ;

- l'absentéisme est suffisamment important pour que l'organisation des équipes et la réalisation du travail soient compliquées, voire problématiques au quotidien dans les écoles.

Cette situation n'est pas nouvelle pour les responsables des Ressources Humaines et du service socio-éducatif dont dépendent les ASEM; elle fait au contraire suite à une série d'événements (mouvement de grève à l'origine de la mise en place de groupes de redéfinition du référentiel des missions des ASEM dans la commune, aménagements du mobilier dans les écoles, formations aux gestes et postures) qui n'ont visiblement pas permis de résoudre les problèmes. Les plaintes des ASEM perdurent, relayées par les représentants des salariés au Comité Hygiène et Sécurité de la commune (CHS). Les responsables de la mairie avouent leur incompréhension vis-à-vis des plaintes d'autant moins audibles pour eux que des concertations et aménagements ont déjà été menés et que les agents d'entretien des écoles élémentaires (aux tâches et conditions de travail comparables aux ASEM de leur point de vue) ne se plaignent pas. Démunie face à cette situation qui provoque des tensions, la mairie souhaite se donner les moyens de comprendre les plaintes et agir.

Dans cette demande, nous trouvons les premiers indices du rôle spécifique du cumul des astreintes dans l'augmentation des pénibilités au travail. Contrairement aux agents d'entretien des écoles élé- 
mentaires, les ASEM ont à assumer des missions éducatives et de restauration en plus de leur mission d'entretien.

Ce cumul de missions, qui conduit à une polyvalence de fait, peut être considéré comme un « enrichissement des tâches » propre à augmenter l'intérêt du travail et à le valoriser tout en créant une alternance et en permettant une certaine souplesse dans son organisation quotidienne. Le risque est que cette organisation multiplie les objectifs à atteindre, les attentes et les directives de part et d'autre, accumule les astreintes de natures diverses inhérentes aux 3 missions, multiplie et diversifie les compétences requises et finalement complique le travail, ne serait-ce qu'en introduisant la nécessité de gérer temporellement des tâches interférentes. Le cumul des missions (la polyvalence induite) pourrait devenir en lui-même une source d'augmentation de la charge de travail, de complication et de pénibilité (Wisner, 1974), à laquelle les agents les plus âgés seraient plus sensibles (Gaudart, 2003).

\section{2.- La situation étudiée}

Les documents existants dans l'institution ont permis de décrire dans un premier temps les tâches à effectuer, l'organisation du travail des ASEM et la population étudiée. En particulier sont utilisés ici : la fiche de poste «ASEM », les bilans sociaux et des données relatives aux ressources humaines (pyramide des âges, maladies professionnelles, accidents du travail, absences, inaptitudes).

\subsection{1.- Une évolution de la fonction d'ASEM vers des missions doubles, voire triples.}

Les prémices de la fonction d'ASEM remontent au XIXème siècle dans le cadre de la création de la fonction de «femme de service» instituée dans des structures d'accueils collectifs de jeunes enfants (Petit, 1994; in Barbier-Le Déroff, 1998). Puis, la fonction d'ASEM glisse officiellement de la réalisation de tâches exclusivement « sanitaires » à deux types de tâches: hygiène et éducation (décret de $1992^{2}$ ). Depuis ce décret, les exigences en matière de compétences obligent les agents occupant la fonction à être titulaires d'un $\mathrm{CAP}^{3}$ " petite enfance » ou parents d'au moins trois enfants (ce qui montre bien l'assimilation du métier au travail de la femme au foyer). Dans la grande majorité des cas, ils sont recrutés sur concours et sont donc des fonctionnaires territoriaux (catégorie C). Toutefois, il peut arriver que des ASEM travaillent sous contrat temporaire en cas de pénurie de personnel ayant obtenu le concours, ou lors du remplacement d'un ASEM titulaire pour congé de longue durée (congé parental par exemple) pour lequel il n'est pas possible de recruter un autre fonctionnaire.

Son statut d'emploi et son lieu d'exercice mettent l'ASEM sous une triple « autorité »: celle formelle de la collectivité qui l'emploie, celle de l'école à laquelle elle est assignée (direction d'école et celle de la direction de centres de loisirs). Elle est en contact au quotidien avec divers acteurs (responsable de la collectivité, direction d'école, enseignants, enfants, personnels périscolaires ou parents) dont les demandes et attentes peuvent être très variées e.g. pour la mairie, l'hygiène des locaux et le service des repas doivent dans tous les cas être assurés; pour les enseignants, l'assistance dans leur activité, l'hygiène des classes et des sanitaires sont essentiels.

Comme l'a constaté Barbier-Le Déroff (1998), les prescriptions relatives à la fonction d'ASEM, et à la définition des tâches ou à leur organisation, présentent des aménagements d'une commune à l'autre. Ainsi, les agents de la mairie de V. remplissent trois missions (au lieu des deux prévues, cf. tableau 1): l'entretien ${ }^{4}$, l'éducation et en plus la restauration. Le décret stipule par ailleurs que les ASEM font formellement partie de la « communauté éducative »; communauté également constituée des enseignants de maternelles et animateurs de centres de loisirs.

2. Décret $n^{\circ} 92-850$ du 28 août 1992 portant statut particulier des cadres d'emploi des ASEM (version consolidée au 29 février 2008), article 2.

3. CAP: Certificat d'Aptitude Professionnelle

4. Depuis 2 ans sont exclues de cette mission les tâches annuelles de manutention du mobilier volumineux et lourd lors des phases de «grand ménage » et les tâches de cirage des sols qui impliquaient des astreintes physiques supplémentaires. Ces tâches sont ici sous-traitées à une entreprise de nettoyage industriel. 


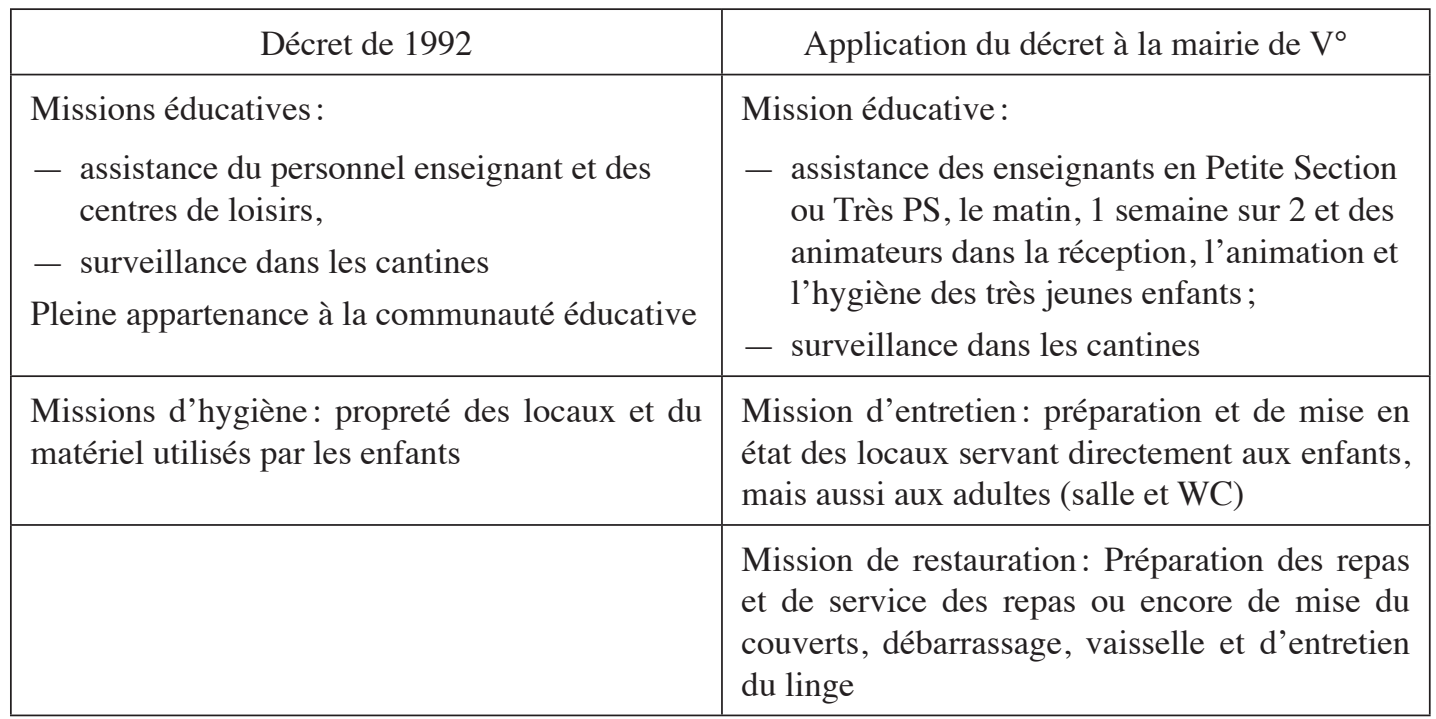

Tableau 1: Comparaison des missions définies par le Décret $n^{\circ} 92-850$ du 28 août 1992 (version consolidée au 29 février 2008) et leur application à la mairie de V.

Table 1: Comparison between the detail of the law on Infant School Assistants' tasks (Décret $n^{\circ}$ 92-850 28 $8^{\text {th }}$ August 1982, modified 29 February 2008) and its application by the V. local authority.

\subsection{2.- L'organisation du travail : une polyvalence à géométrie variable}

Dans cette municipalité, les ASEM sont entre 4 et 10 par écoles, en fonction du nombre de classes dans l'école où elles sont affectées; ce nombre peut donc évoluer d'une année sur l'autre en fonction des ouvertures ou fermetures de classes. Les ASEM titulaires sont affectées, sauf exception (accident ou maladie nécessitant une réaffectation), à une école pour l'année scolaire. Parmi les critères d'affectation, une attention particulière est donnée au fait qu'il n'y ait pas plus de 3 personnes avec une restriction médicale par école, ce qui peut générer des variabilités dans les caractéristiques des tâches des autres personnes d'une équipe.

Afin de couvrir la plage d'accueil des enfants, deux horaires de travail sont pratiqués : $7 \mathrm{~h} 30$ - 14h 30 (équipe du matin) et $10 \mathrm{~h} 00-17 \mathrm{~h} 00$ (équipe de l'après-midi). L'équipe d'ASEM étant scindée en deux, l'effectif est au complet seulement durant la période de recouvrement $(10 \mathrm{~h}-14 \mathrm{~h} 30)$, c'est-àdire 4 heures par jour. Les ASEM alternent sur ces deux horaires une semaine sur deux.

Les remplacements ne sont effectifs qu'en cas d'absence simultanée d'au moins 2 ASEM sur l'ensemble des personnes affectées à une école. Ils sont assurés par des ASEM ou des adjoints techniques non titulaires (affectés dans le cas d'absences longues et prévisibles) ou des agents d'entretien horaires vacataires (affectés lors d'absences courtes et qui accomplissent exclusivement les tâches d'entretien et de restauration).

Compte tenu des trois missions à accomplir, de l'alternance des horaires de travail et du remplacement non systématique des agents absents, l'organisation prescrite et la gestion effective du travail repose sur la polyvalence des ASEM en poste. Parmi les tâches à accomplir, certaines sont prévisibles et peuvent donc être anticipées (tâches d'entretien et de restauration), d'autres sont plus aléatoires, voire imprévisibles (hygiène auprès des enfants, assistance des enseignants en petite section). La polyvalence et le caractère imprévisible de certaines tâches accroissent le risque de tâches interférentes à gérer dans l'action. 


\subsection{3.- La population : diversité quant à l'âge et l'état de santé}

L'étude des bases de données du service des Ressources Humaines confirme les informations fournies par la mairie quant à la répartition des âges, le volume général de jours d'arrêts pour raison de santé ou accident du travail et de déclaration des maladies professionnelles. Elle permet de les affiner en montrant que:

- la population est essentiellement féminine dans cette commune (seulement 2 hommes font partie de l'effectif $)$;

- âgées de 19 à 62 ans, 157 ASEM (Titulaires ou adjointes techniques) occupent cette fonction, sur 142 postes et demi. L'année de l'étude, $57 \%$ d'entre elles ont plus de 40 ans. Elles ont de quelques mois à 38 ans d'ancienneté dans cette mairie;

- les ASEM recrutées avant 1992 (date de décret régissant la fonction) n'ont pas toutes le CAP «petite enfance », même si certaines l'ont acquis dans le cadre de la $\mathrm{VAE}^{6}$; celles recrutées par la suite ont obligatoirement ce diplôme. On a donc ici un premier effet de génération;

- les plus de 40 ans ont un peu plus de journées d'absences que les plus jeunes (46,1\% contre $43,9 \%)$;

- le nombre moyen de journées d'absence (8 jours/an en moyenne) évolue peu jusqu'à 49 ans puis augmente fortement (17j/an pour les plus de 50 ans);

- parmi celles qui s'arrêtent, les moins de 40 ans ont moins d'arrêts pour accident du travail que les plus âgés et s'arrêtent moins longtemps (26 jours en moyenne pour les plus de 50 ans et 8 jours en moyenne pour les moins de 40 ans);

- par ailleurs, en 3 ans : 34 restrictions médicales définitives ou temporaires et 2 inaptitudes ont été déclarées (10 à 15/an répartis sur 22 ASEM en tout). Le nombre de restrictions et d'inaptitude est nettement plus élevé chez les plus de 40 ans (28/36). Les restrictions sont plutôt temporaires chez les moins 40 ans (5/6) et majoritairement permanentes chez les plus âgés $(20 / 28)^{7}$.

Les premiers indicateurs de santé (absences, inaptitude, restrictions médicales), tout relatifs qu'ils puissent être, laissent apparaître un effet d'âge ou un second effet de génération (lié aux conditions de travail passées) puisque les ASEM plus âgées ont des indicateurs moins favorables que leurs cadettes. En se référant à la notion de «pénibilités » retenue plus haut, nous pouvons avancer ici que l'état de santé déficient des ASEM de 40 ans et plus accroîtra en retour les pénibilités au travail en induisant des gênes et difficultés dans l'accomplissement de leurs missions.

\section{4.- Des techniques d'investigation qualitatives et quantitatives}

\section{1.- La démarche générale d'investigation}

La démarche privilégiée est une approche participative (Rogard, 1997). Un groupe de pilotage a été constitué et a fonctionné sur la durée de l'étude (1 an). Il regroupait des représentants des services des Ressources Humaines et Socio-Educatif dont dépendent les ASEM, deux élus de la mairie, le médecin et l'infirmière du travail d'un service inter-entreprise dont dépend la mairie, la psychologue du travail de la mairie, trois représentantes des ASEM et un du CHS afin de permettre à tous de participer aux choix faits durant l'étude et d'être informés de son avancée.

5. C'est pour cette raison que nous employons le féminin pour parler des ASEM.

6. VAE: Validation des Acquis de l'Expérience

7. On trouve ici une population presque exclusivement féminine et plutôt âgée, comme dans les travaux menés dans les secteurs de la restauration collective et du nettoyage. Toutefois le statut de fonctionnaire territorial, obtenu par concours et conditionné à l'obtention d'un diplôme, place ces personnes dans des conditions salariales durables contrairement à la plupart des employées des deux autres secteurs. 
En revanche, ni les enseignants ni les animateurs n'ont pu participer à ce groupe, la Direction Générale de la mairie s'y opposant au motif que les premiers n'étaient pas des fonctionnaires territoriaux et que les seconds étaient déjà représentés par le responsable du service socio-éducatif. Face aux arguments contraires fournis par l'équipe de recherche, il a toutefois été admis que ces principaux protagonistes du travail des ASEM pourraient être sollicités dans le cadre d'entretiens afin de recueillir leurs points de vue sur la situation étudiée.

Cette recherche a été réalisée sur la base du volontariat et dans le respect de l'anonymat de chacun.

\section{2.- Les outils de recueil des données}

Trois types d'outils de recueil ont été utilisés.

\subsection{1.- Des entretiens exploratoires}

14 entretiens exploratoires ont été réalisés auprès de différents acteurs de la mairie et des écoles, susceptibles d'être en contact avec les ASEM, et auprès des agents eux-mêmes en dehors de leur activité de travail.

Selon la démarche d'investigation proposée par Guérin, Laville, Daniellou, Duraffourg et Kerguelen (1997), ces entretiens ont eu pour but de décrire le rôle de chacun, ses caractéristiques, ainsi que les liens qu'ils entretiennent entre eux. Ils ont également permis de se familiariser avec le fonctionnement de l'institution et la situation des ASEM, d'identifier des écarts entre le prescrit défini dans le décret de 1992 et sa déclinaison au sein de la mairie, de lister les tâches relevant de chaque mission. Une facette de la pénibilité, le vécu difficile de l'activité professionnelle, a également pu être appréhendée de cette manière.

Afin de préciser et d'enrichir ces premières analyses, l'ensemble des éléments ainsi identifiés a servi à la construction d'indicateurs de l'activité dans le cadre des observations systématiques, mais aussi la rédaction d'items d'un questionnaire relatifs à l'organisation du travail, à la perception des conditions d'exercice et de leur santé.

\subsection{2.- Des observations systématiques de l'activité}

Comme il n'était pas possible d'observer toutes les ASEM travaillant dans les 24 écoles maternelles de la ville, des critères de choix d'écoles ont été définis à partir des entretiens et de l'analyse quantitative des données démographiques, d'absences, de maladies professionnelles et d'accidents du travail recueillies auprès du service des Ressources Humaines. Ceci a permis de catégoriser les écoles en fonction de:

- la taille de l'école et des effectifs d'ASEM: de 4 à 10 ASEM par école;

- la physionomie d'âge de l'équipe de chaque école: a) plutôt jeune, b) plutôt âgée, c) variée;

- les Accidents du Travail (AT), Maladies Professionnelles (MP), Maladies Ordinaires (MO) et restrictions déclarées entre 2005 et 2007 par chaque personne: a) un cumul d'absences pour MP et/ou AT, b) des AT ou MP mais relativement peu d'absences, c) des absences rares et pas d'AT et MP sur la période considérée.

Le choix a ensuite tenu compte de deux contraintes: a) limiter le nombre d'écoles à 3 par souci de faisabilité des investigations dans le temps imparti (2 mois et demi de présence dans les écoles); b) limiter la diversité des situations étudiées aux seules conditions d'âge, de santé et d'absentéisme, en ciblant des écoles dont les équipes étaient composées sensiblement du même nombre d'ASEM (i.e., les équipes de 5 ASEM qui sont les plus nombreuses).

Dans le cadre de contraintes, les trois écoles retenues ont les caractéristiques suivantes: l'école A: une majorité d'ASEM âgées de plus de 40 ans, dont le nombre d'AT, de MP et d'absences est élevé par rapport aux autres écoles; l'école B : des ASEM d'âges variés, certaines ayant eu des AT, mais 
pas beaucoup d'absences par rapport aux autres écoles; et l'école C: une majorité d'ASEM âgées de plus de 40 ans, n'ayant jamais eu d'AT ou MP depuis 2005 et très peu d'absences. Pour chaque école, nous avons obtenu l'accord de la Direction du service socio-éducatif, des Directions d'écoles ou de Centres de Loisirs Maternels et des ASEM y travaillant.

À partir de 7 observations ouvertes de l'activité en période scolaire, nous avons défini puis validé auprès du comité de pilotage ce que recouvrent les différentes missions comme suit:

- «entretien » qui recouvre: le nettoyage des locaux (classes, réfectoire, dortoir, toilettes, cuisine, salle polyvalente, salle des enseignants) et du matériel (tables, chaises, pinceaux, tableaux, etc.); le rangement (des classes, de la vaisselle, etc.); la vaisselle; le changement des corbeilles et poubelles; le lavage, séchage et le pliage du linge;

— « restauration » où l'on trouve: le décartonnage des aliments conditionnés (plats cuisinés, pots de yaourts, etc.), la vérification de la température de conservation des plats, la préparation de la collation, le lavage des aliments frais et leur découpe (légumes, fruits, pain), le dressage des plats, la mise en chauffe, la mise du couvert, le service des plats à chaque table, le débarrassage;

— «éducation » qui a lieu en présence d'un enseignant ou animateur et concerne: l'aide à l'habillage; au déshabillage et au passage aux toilettes des enfants; la préparation ou l'animation d'un atelier en classe; l'assistance aux enfants en classe (accueil et surveillance), la surveillance des enfants au réfectoire et l'aide à table (pour finir une assiette, découper en morceaux, peler un fruit, etc.).

Sur cette base, 10 observations systématiques ont été réalisées. Nous avons relevé la nature, la fréquence d'occurrence et la durée des tâches précédemment identifiées et d'autres éléments permettant de caractériser plus finement l'activité des ASEM:

- le lieu d'exécution de la mission (classe, toilettes, réfectoire, dortoir, salle des enseignants, cuisine, etc.) pour la reconstitution des déplacements au cours du travail;

- la présence d'autres personnes dans le lieu de travail (enfants, enseignants, directeur d'école, animateurs, parents, ASEM) et les communications avec les acteurs précédemment cités en vue d'appréhender des dimensions collectives du travail;

- l'adoption de postures reconnues pour la plupart comme pénibles dans la littérature scientifique: Debout penché, debout plié, assis (chaise d'enfant ou d'adulte), assis penché, assis plié, à genoux ou accroupi; et le port de charges : seau, chaise, pile d'assiettes, bac de verres, de gobelets ou de couverts, poubelle de réfectoire, corbeille, panière à linge, cagette de fruits et légumes frais, enfant, chariot; leur poids s'étendant de $2 \mathrm{~kg}$ (e.g. un bac de verre) à $17 \mathrm{~kg}$ (e.g: une poubelle de réfectoire): autant d'indices des pénibilités physiques du travail.

L'ensemble des relevés systématiques a été traité à l'aide du logiciel «Actogram-Kronos » (Kerguelen, 2008). Les analyses descriptives menées sur cette base permettent de comprendre comment les 3 missions et leurs tâches afférentes s'enchaînent, s'articulent, s'entrecroisent dans le temps. Elles permettent de rendre compte des ressources ou des astreintes physiques et psychologiques inhérentes aux missions et de l'exposition aux conditions de travail reconnues dans la littérature comme potentiellement pathogènes à plus ou moins long terme. Elles permettent ainsi de repérer des symptômes d'astreintes psychologiques aidant à faire des liens entre un vécu difficile ou une santé déficiente et les conditions de travail; ce qui constitue une autre dimension des pénibilités au travail.

\subsection{3.- Un questionnaire « santé - travail »}

Comme nous l'avons déjà dit, dans le temps imparti pour la recherche, il n'était possible d'observer ni toutes les ASEM ni la totalité des situations de travail existantes. Pourtant il était nécessaire d'avoir une connaissance plus étendue de la diversité des situations, des conditions de travail et des représentations que se faisaient les ASEM de leur activité et de leur état de santé. C'est pourquoi nous 
avons élaboré un questionnaire sur les conditions de travail et la santé perçue, dans le but de pouvoir estimer l'importance de dimensions sur lesquelles l'observation de l'activité avait attiré notre attention et d'inventorier leurs éventuelles conséquences (Prunier-Poulmaire \& Gadbois, 2005).

Intitulé « parcours professionnels, exigences du travail et santé des ASEM », ce questionnaire a été construit pour partie en reprenant des outils déjà existants, jugés pertinents pour les situations observées (le questionnaire du Réseau expérimental de surveillance épidémiologique dans le pays de Loire et l'enquête SVP50 20038; Molinié, 2005; Ha \& Roquelaure, 2007). Des questions spécifiques y ont été ajoutées. L'ensemble a été validé au cours d'une réunion du groupe de pilotage. Afin de s'assurer de la participation d'un nombre important d'agents, et compte tenu de la longueur du questionnaire (87 questions), il a été convenu que sa passation se ferait en deux phases: a) une réunion de présentation globale auprès de l'ensemble des ASEM, en présence des responsables du service Socio-Educatif; b) la passation en salle, guidée si nécessaire par l'ergonome présent. Afin de respecter l'anonymat des personnes présentes à cette deuxième réunion, il a été convenu que les responsables du service Socio-Educatif n'y assisteraient pas. Le questionnaire rempli était remis en séance au chercheur. Le taux de réponses a été de $56,7 \%$ de la population générale.

Dans cet article, les items traités ont été choisis en fonction de leur pertinence par rapport à l'identification des pénibilités. Ces items se répartissent sous les thèmes suivants:

- situation personnelle (âge, sexe, statut d'emploi, obtention du CAP petite enfance);

- conditions de travail (horaires et temps de travail, conditions physiques et organisation du travail);

— évaluation subjective du travail (pénibilité physique, intérêt, complication, moyens pour faire son travail...);

- état de santé perçu (état général, troubles infra-pathologiques, douleurs et TMS).

Les réponses se font sous plusieurs modes: la plupart des questions consistent à se positionner sur une échelle d'accord (type échelle de Lickert; Pensez-vous que, dans l'ensemble, vous avez le temps de faire un travail de qualité? jamais, rarement, souvent, toujours). D'autres supposent une réponse binaire (Aimeriez-vous participer davantage au projet d'école? oui non), ou à choix multiple (Compte tenu de votre activité générale, estimez-vous que les respect des règles d'entretien des locaux est réalisable? oui/non. Si non, est-ce parce que: vous manquez de temps, la charge de travail est trop importante, les règles sont en contradiction avec d'autres règles que vous devez suivre, etc.).

Une fois recueillies, les réponses ont été numérisées et traitées à l'aide d'un tableur sur la base d'analyses descriptives (fréquences d'occurrence, durées d'état).

\section{5.- Des astreintes physiques différenciées qui se cumulent}

L'analyse des 10 relevés d'observations et des entretiens avec les ASEM offre l'opportunité d'identifier comment les trois missions et les tâches sous jacentes se déroulent et s'articulent tout au long de la journée de travail. Deux points sont retenus : la disparité du poids des missions et les pénibilités physiques avérées dans ce travail.

\section{1.- Disparité du poids des missions}

Ces analyses permettent de montrer que les missions d'entretien, de restauration et d'éducation n'ont pas le même poids dans l'activité quotidienne des ASEM. Cette disparité est liée à diverses variables de situation:

8. Santé et Vie Professionnelle après 50 ans (SVP50). (2003). Enquête réalisée par le Centre Interservices de Santé et de Médecine du travail en Entreprise (CISME), http://www.cisme.org/. 
- individuelles: l'état de santé de l'ASEM observée et/ou de celui des collègues (des restrictions médicales temporaires ou permanentes); le statut d'emploi qui ne permet pas toujours de réaliser la mission éducative dans les classes lorsque qu'elles sont adjoints techniques;

- météorologiques, saisonnières ou calendaires qui induisent plus ou moins de travail d'entretien (pluie, neige $v s$ soleil) ou éducatif (guidage des enfants plus important en début d'année; aide à l'habillage plus long en hiver; préparation de fêtes annuelles locales ou nationales);

- de degré d'autonomie des enfants auxquels l'ASEM est affectée durant le repas (aide à découper les aliments, ou à manger);

- des événements ponctuels : anniversaires d'enfants, ateliers de cuisine ou d'activités salissantes (peinture), sorties qui vont ajouter des tâches et réorganiser celles initialement prévues;

- organisationnelles: les règles de remplacement dans les équipes (les ASEM observées travaillent en sous-effectif dans 5/10 journées étudiées) et la priorité donnée par la mairie aux missions d'entretien et de restauration d'une manière générale (les ASEM n'assistent les enseignants que dans les classes de petites sections lorsqu'elles font partie de l'équipe du matin) ou en cas de sous-effectif (où l'assistance aux enseignants peut être abandonnée momentanément); l'organisation de l'équipe spécifique à chaque école (dans la répartition des tâches); le roulement entre les équipes horaires.

Ces variables pèsent fortement sur la répartition et le poids relatif de chaque mission puisque l'analyse descriptive en durée et en fréquence d'occurrence des tâches montre que le matin ce sont les missions d'éducation et d'entretien qui sont majoritaires (respectivement 42,5\% et 37,7\% du temps) alors que l'après-midi les missions d'entretien et de restauration priment (55,8 \% et 25,6\% du temps).

Par ailleurs, dans $41 \%$ du temps qui lui est consacré, la mission d'éducation est souvent effectuée en simultané avec des tâches d'entretien et surtout de restauration. En horaire d'après-midi, la mission éducative, d'une durée moins importante d'une manière générale, se fait principalement dans des conditions de cumul de missions (60\% du temps contre $37 \%$ le matin; cf. Figure 1). Une ASEM va, par exemple, nettoyer la peinture tombée d'un pot tout en continuant à « animer » un atelier dans la classe; une autre tentera d'aider un groupe d'enfants durant le repas tout en préparant les fruits prévus en désert (éplucher et découper le fruit de chaque enfant).

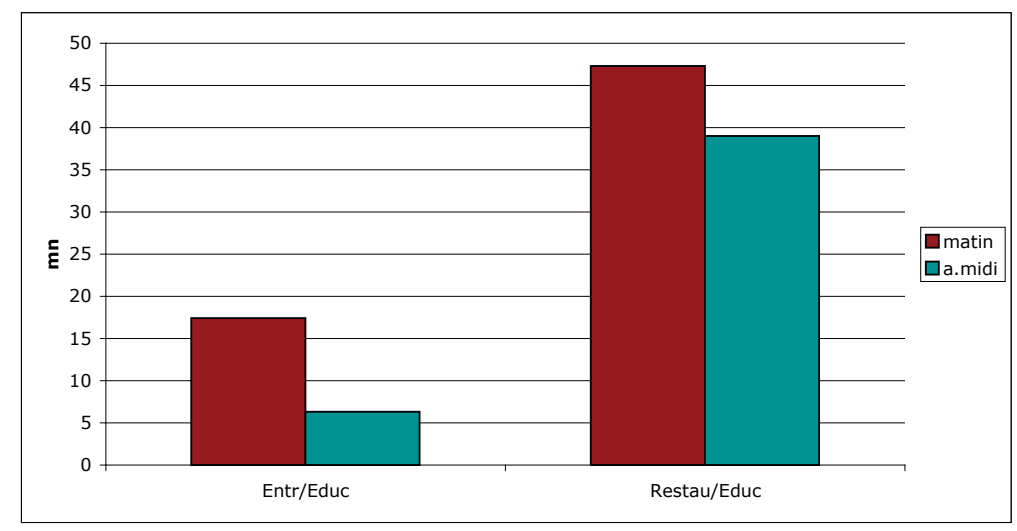

Figure 1: Temps (en minutes) pendant lequel la mission d'éducation (Educ) est réalisée en simultané avec les missions d'entretien (Entr) ou de restauration (Restau) suivant les postes du matin ou de l'après-midi.

Figure 1 : Time spent (in minutes) on educational tasks while simultaneously carrying out cleaning or catering tasks in the morning and afternoon

Ceci laisse supposer un amoindrissement de la mission éducative essentiellement consacrée à des tâches ponctuelles de « régulation », de « modération » plutôt que d'éducation (assistance des enseignants en classe ou des animateurs dans le réfectoire qui se limite au rappel des règles de vie en groupe). 


\section{2.- Des risques encourus variables avec les missions}

L'analyse des comptes rendus d'Accidents du Travail (AT) durant les 2 années précédant l'étude souligne que les trois missions n'ont pas les mêmes conséquences en termes de sécurité.

Les 35 AT déclarés, peuvent être catégorisés en 3 grands types:

- les « blessures » (17/35), par coupure (les tableaux où l'on se coupe les doigts), piqûre, contusion (les lits et matelas avec lesquels on se coince les doigts), écrasement, projection ou brûlure en cuisine;

- les « chutes » (7/35) de plain-pied (glissade sur sol mouillé), ou en dénivelé (monter/descendre marche, avec éventuellement port de charge);

- les «douleurs» (7/35) qui apparaissent lors de la manipulation de charges et objets encombrants, du rangement dans des meubles très bas adaptés à la taille des enfants, d'allergies, de mauvaise tenue du pied dans les sabots, ou de déséquilibre.

Par rapport à l'âge, ces accidents sont légèrement plus fréquents chez les ASEM de plus de 40 ans (19 concernent des +40 ans et 16 AT des -40 ans).

Des différences plus marquées apparaissent entre les missions : les AT ont lieu essentiellement durant la mission d'entretien (17/35) et dans une moindre mesure au cours des missions de restauration (9/35) et d'éducation (3/35; cf. Figure 2). Il est regrettable que les circonstances de 6 accidents ne soient pas renseignées ce qui suppose qu'ils n'ont pas fait l'objet d'analyse dans l'institution.

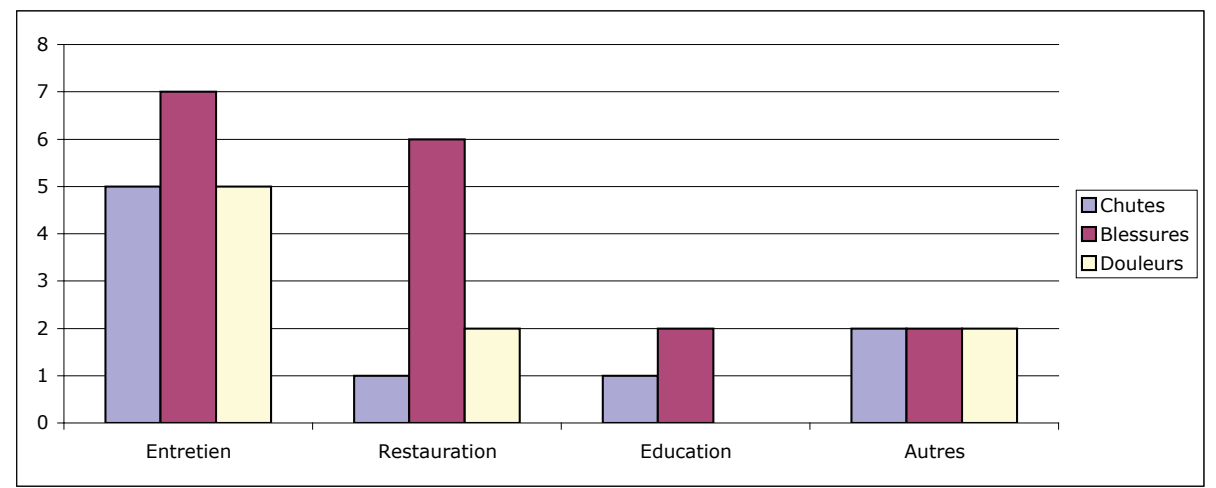

Figure 2: Fréquence et types d'accidents du travail (chutes, blessures, douleurs) survenus dans le cadre des missions d'entretien, de restauration, d'éducation ou autres (non renseignées ou à l'entrée de l'école) durant les deux années précédant l'étude.

Figure $2:$ Rates and types of accidents at work (falls, injuries, pain) caused by cleaning, catering or classroom tasks (or "other" unknown tasks) over the last two years.

Ces différents AT témoignent de l'inadéquation de certains outils ou matériels (sabots, tableaux, lits, meubles de rangement, grilles de four, etc.) et de l'existence de situations où le travail est réalisé sous contraintes temporelles et/ou physiques fortes avec absence momentanée de possibilité d'entraide: chutes de plain-pied ou en dénivelé avec port de charge, blessures par compression.

\section{3.- Des conditions de travail pénalisantes : déplacements, postures et port de charges}

L'analyse des lieux d'exercice, des postures et des ports de charges offre la possibilité de traiter des pénibilités physiques du travail.

D’une manière générale, les ASEM occupent 10 lieux différents dans une journée de travail. Elles y séjournent plus ou moins longtemps et 3 lieux se distinguent nettement par la durée d'occupation: les classes (Moy. 1 h), le réfectoire (Moy. 1 h 20 min) et la cuisine (Moy. 1 h $14 \mathrm{~min}$ ). On note éga- 
lement une répartition distincte des lieux de travail entre le poste du matin et celui de l'après-midi. Les ASEM du matin sont davantage dans les classes ou à l'office (pour le pliage du linge) que celles de l'horaire d'après-midi (Moy. classe $1 \mathrm{~h} 15$ contre 30min; Moy. Office $34 \mathrm{~min}$ contre $4 \mathrm{~min}$ ) alors que les ASEM de l'après-midi sont plus longtemps en cuisine (Moy. $1 \mathrm{~h} 52 \mathrm{~min}$ contre $57 \mathrm{~min}$ ). Cette différence est liée au type de mission qu'accomplissent les ASEM: davantage éducative le matin et en grande partie de restauration ou d'entretien l'après-midi.

Les fréquences de présence dans les différents lieux montrent en contrepoint les nombreux déplacements des ASEM au cours de la période de travail. Ils peuvent être ramenés à 1 déplacement toutes les 11 à 13 min en moyenne sur $7 \mathrm{~h} 30 \mathrm{~min}$ de travail; ces déplacements se faisant dans des écoles plus ou moins grandes (et donc sur des distances plus ou moins importantes) et parfois à étages (ce qui suppose l'utilisation plus ou moins fréquente d'escaliers). Par ailleurs, deux lieux semblent souvent fréquentés (d'autant plus en poste d'après-midi): le réfectoire et la cuisine, attestant d'un va-etvient entre ces deux lieux notamment durant la période de midi.

Les postures « debout », « debout penché » sont les plus fréquentes durant les vacations du matin et de l'après-midi; elles sont adoptées par défaut. Étant donné le nombre important de déplacements, les ASEM piétinent donc toute la journée. Elles portent ou transportent 25 charges en moyenne sur la durée d'une vacation (de $2 \mathrm{~kg}$ à $17 \mathrm{~kg}$; il peut aussi bien s'agir des enfants, des casiers des vaisselles, de panières de linge que de sacs poubelles). Elles ont à produire des efforts dynamiques sur de courtes durées dans un environnement au mobilier bas adapté à la taille des enfants.

Référées à la catégorisation définie à partir de l'enquête SUMER 2003 (Arnaudo, Hamon-Cholet \& Waltisperger, 2006), les astreintes physiques mises en évidence dans cette situation sont des contraintes «lourdes » qui augmentent fortement la probabilité d'avoir des problèmes de santé, des TMS en particulier. Ce sont notamment:

— des « situations fatigantes »: maintien de postures « debout » ou «debout penché » plus de $20 \mathrm{~h}$ par semaine; des déplacements et piétinements (+ de 20h/ semaine);

— des «postures pénibles »: être accroupi, à genoux (+ de $2 \mathrm{~h} /$ semaine) en raison du mobilier bas, peu adapté aux adultes et à l'activité des ASEM;

- des ports de charges conséquents (jusqu'à $20 \mathrm{~kg}$ ).

Par ailleurs, les fréquents déplacements entre lieux dans les écoles (le réfectoire et la cuisine par exemple) attestent des difficultés de mise en œuvre de la mission éducative sur le temps de midi.

\section{6.- Des symptômes de pénibilités et de mal-être au travail}

L'analyse des réponses fournies au questionnaire « santé-travail » a pour but de dresser un tableau des troubles infra-pathologiques perçus et des représentations que les ASEM se font de leurs conditions de travail. Elle permet d'avoir une idée du confort, de la satisfaction au travail ou inversement des difficultés et des sources de pénibilités ressenties par les personnes interrogées.

\section{1.- Une multiplicité de troubles infra-pathologiques}

D’une manière générale, $78 \%$ des ASEM de moins de 40 ans se considèrent en « bonne » santé; elles sont toutefois un peu plus nombreuses à penser que celle-ci s'est dégradée ces dernières années (53\%) plutôt que restée stable (47\%). Les plus 40 ans sont autant à se considérer en « bonne » $(51 \%)$ qu'en « mauvaise » santé $(49 \%)$ et $59 \%$ disent que leur santé s'est dégradée.

L'analyse des déclarations de troubles infra-pathologiques (Molinié et Volkoff, 2002) montre que les ASEM se sentent majoritairement:

— fatiguées: «troubles de sommeil » $(65 \%)$ quel que soit leur âge et « sensation de fatigue » ou

9. SUMER: SUrveillance MÉdicale des Risques professionnels. 
« difficultés à récupérer » pour respectivement $100 \%$ et $60 \%$ des femmes de plus de 40 ans;

- abîmées: «douleurs » (87 \%) quel que soit l'âge, et «difficultés dans les mouvements ou gestes » pour $68 \%$ des plus de 40 ans;

- découragées: « sentiment de découragement » pour $52 \%$ des plus de 40 ans.

Dans au moins $70 \%$ des cas où elles déclarent un trouble, jeunes et âgées considèrent qu'il les gêne dans le travail.

Ces chiffres sont plus élevés que ceux obtenus dans l'enquête SVP 50 (2003) où, par exemple, seulement $19 \%$ des femmes âgées de 50 ans et plus s'estimaient en mauvaise santé et considéraient l'évolution de leur santé comme stable dans $61 \%$ des cas, $35 \%$ dégradée, $4 \%$ améliorée. On retrouve des tendances similaires pour la plupart des troubles infra-pathologiques (cf. Figure 3), ce qui appuie l'hypothèse des effets délétères des astreintes physiques et psychologiques avec lesquelles les ASEM doivent composer.

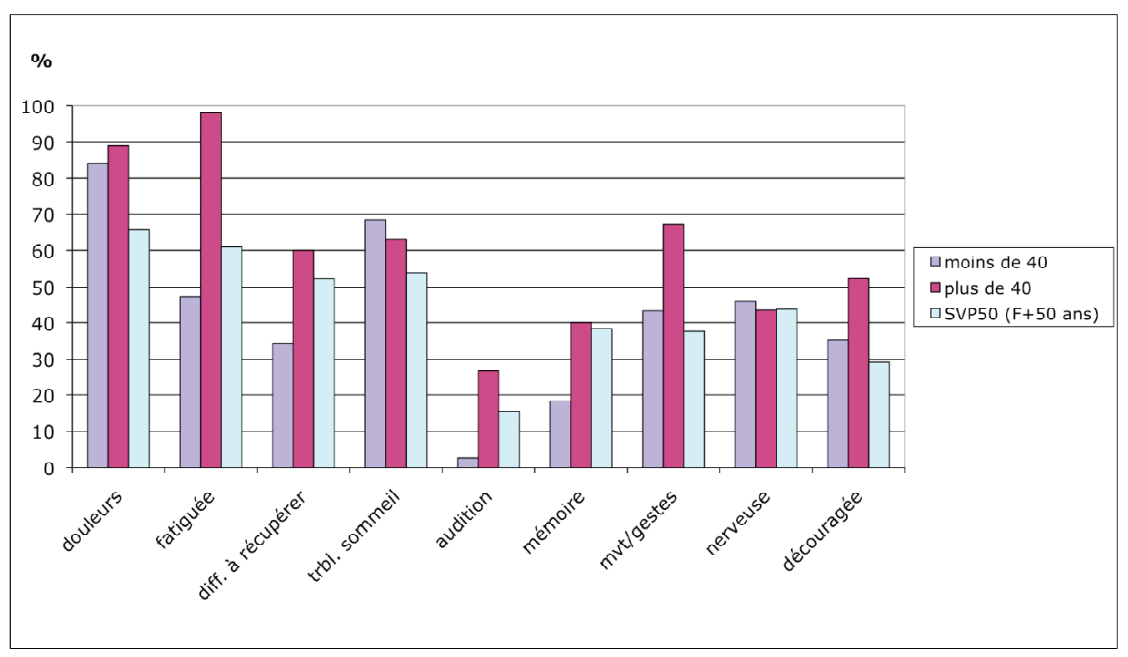

Figure 3: Répartition (en \%) des troubles infra-pathologiques perçus par les ASEM de moins et de plus de 40 ans, et par les femmes de plus de 50 ans issues de l'enquête SVP50.

Figure 3: Percentage of infra-pathological disorders reported by ISAs under 40 years old, ISAs over 40 years old, and by women of 50 years and over (SVP50 survey).

Ces résultats soulignent l'évolution des pénibilités liées à une santé déficiente avec l'avancée en âge : les ASEM âgées étant plus nombreuses à déclarer des troubles, ressentent également plus fréquemment des gênes dans leur travail. Ils laissent également supposer que si les plus jeunes se considèrent en grande majorité en "bonne santé », certaines pourraient avoir une impression d'être déjà dans un processus de déclin puisqu'elles sont une majorité à répondre que leur santé s'est dégradée durant les dernières années.

\section{2.- Missions et conditions de travail : des sentiments à double titre «partagés »}

Dans l'ensemble, les impressions concernant leurs conditions de travail sont mitigées.

Si $60 \%$ des ASEM considèrent être suffisamment informées pour effectuer leur travail et $61 \%$ être soutenues par leur hiérarchie, $50 \%$ des moins de 40 ans et $70 \%$ des plus âgées estiment ne pas disposer de moyens matériels et humains suffisants. $52 \%$ pensent également ne pas avoir le temps de faire un travail de qualité et $62 \%$ ne pas être reconnues en général dans leur travail, ceci quel que soit leur âge.

Les missions d'entretien, de restauration et d'éducation ne sont pas perçues de façon similaire: 
- l'entretien est perçu comme pénible par beaucoup contrairement à l'éducation ;

- les résultats s'inversent lorsque les ASEM se positionnent sur l'intérêt de ces mêmes missions ;

- aucune différence selon l'âge n'est observée.

Ces représentations corroborent les analyses des accidents du travail où la mission d'entretien était identifiée comme celle des trois missions associée à davantage de risques.

Les ASEM jeunes et âgées ont donc une perception doublement partagée de leurs conditions de travail. Bien qu'elles se sentent dans l'ensemble soutenues et suffisamment informées, elles considèrent majoritairement ne pas être reconnues et ne pas pouvoir faire un travail de qualité. Par ailleurs, alors que l'on peut constater des différences en fonction de l'âge sur la santé perçue, elles partagent des jugements mitigés sur les caractéristiques des trois missions.

\section{3.- Une intégration sociale limitée}

D'une manière générale, on considère que les relations sociales au sein d'un collectif de travail permettent de développer des aides et des solidarités qui permettent d'atténuer les difficultés rencontrées dans le travail (Cau-Bareille, 2007). Ici, l'analyse de la dimension collective du travail est objectivée pour partie par les relevés de durée et fréquence de travail seule ou en présence d'une ou plusieurs personnes (adultes et/ou enfants), et le décompte des communications en fonction des interlocuteurs (collègues, enseignants, animateurs, enfants, parents). S'y ajoute le traitement de 3 items du questionnaire relatifs à l'auto-évaluation de l'autonomie dans le travail. Bien qu'un peu schématique, cette analyse permet de montrer un isolement relatif des ASEM.

\subsection{1.- Un travail essentiellement en co-présence}

Tout au long de la journée, les ASEM travaillent le plus souvent en co-présence d'une ou plusieurs personnes ( $1 \mathrm{~h} 12 \mathrm{~min}$ seule en moyenne sur $7 \mathrm{~h} 30$ de présence). Parfois en binôme avec une autre collègue (Moy. $1 \mathrm{~h} 36 \mathrm{~min}$ ), elles sont le plus fréquemment en présence des enfants (Moy. $2 \mathrm{~h} 18 \mathrm{~min}$ ) sous l'autorité de différentes personnes : enseignant, animateur, ou directrices de l'école ou du centre de loisirs.

Le matin, les ASEM travaillent autant seules (Moy. $1 \mathrm{~h} 17 \mathrm{~min}$ ) qu'en binôme (Moy. $1 \mathrm{~h} 19 \mathrm{~min}$ ). Elles sont principalement en co-présence des enfants (Moy. 2 h) sous l'autorité des enseignants

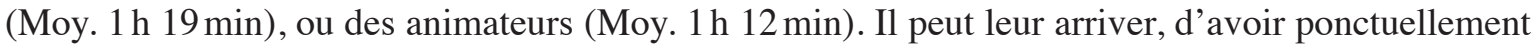
la responsabilité des enfants (seules avec les enfants ou à deux, Moy. 13 min).

L'après-midi, les ASEM travaillent seules (Moy. 1 h) et surtout en binôme (Moy. $2 \mathrm{~h} 18 \mathrm{~min}$ ).

Elles ont moins de contact avec les enfants (Moy. $1 \mathrm{~h} 23 \mathrm{~min}$ ) et les différents acteurs de l'école (10 min en moyenne avec un enseignant) ou du centre de loisirs ( $1 \mathrm{~h}$ en moyenne avec le personnel d'animation). Quasiment aucun contact avec les parents n'a été constaté pendant les périodes d'observation.

Cependant, cette co-présence ne signifie pas forcément collaboration; on pense plutôt à une co-action $^{10}$, si l'on en juge par les échanges verbaux observés.

\subsection{2.- Des échanges verbaux peu nombreux}

Les ASEM ont différents interlocuteurs potentiels (ceux avec lesquels elles travaillent en co-présence exclusivement, cf. Figure 4).

10. «La co-action concerne des situations dans lesquelles les opérateurs poursuivent des actions différentes sur des objets différents en ayant des buts à court terme différents mais devant être intégrés à moyen terme dans une activité commune. La collaboration caractérise une activité collective dans laquelle les opérateurs accomplissent sur un même objet des opérations différentes qui s'articulent les unes aux autres avec un but commun à court et à moyen terme. » (De la Garza \& Weill-Fassina, 2000,p. 227) 
- leurs principaux interlocuteurs sont les enfants avec lesquels elles parlent en moyenne 91 fois par vacation de $7 \mathrm{~h} 30$, des enfants qui leur sont directement affectés lors de l'animation d'un atelier et de l'aide à table ou d'enfants qu'elles surveillent (en classe, au réfectoire); ce qui correspond à leur fonction éducative;

- viennent ensuite leurs collègues (Moy. 10 fois) et les enseignants (Moy. 9 fois). Elles communiquent plus rarement avec les animateurs même si elles travaillent avec eux au moins pendant le temps de repas. Ceci représente des coordinations et des concertations très limitées au cours du travail;

- enfin, les échanges avec les parents sont exceptionnels. Ces contacts ne sont pas prévus dans leur fonction.

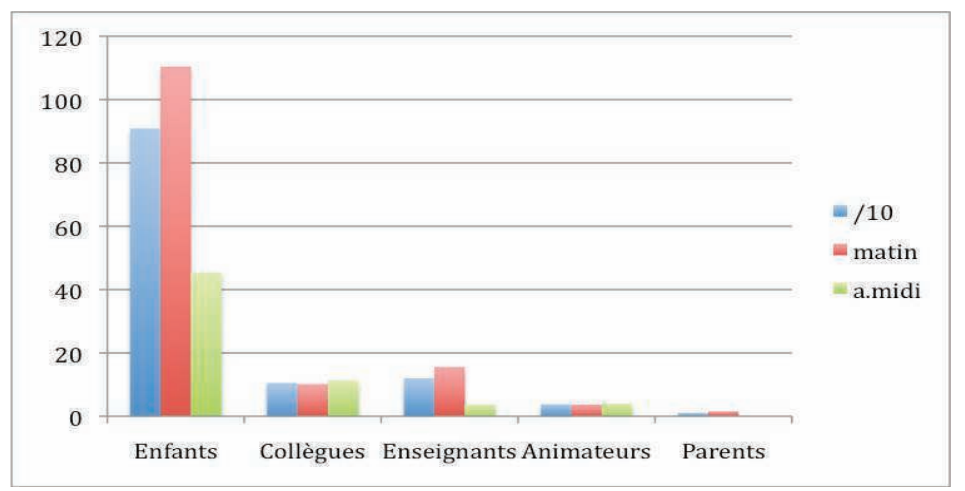

Figure 4 : Fréquences moyennes des échanges verbaux avec différents acteurs de l'école ou du centre de loisirs: a) sur les 10 relevés d'observation; b) lors du poste du matin; c) l'après-midi.

Figure 4: Mean frequency of discussions with different people in the schools or play centers during the 10 observation sessions, in the morning, and in the afternoon, split between discussions with children, colleagues, teachers, activity leaders, and parents.

Les ASEM ne parlent aux enfants qu'en présence d'autres adultes et ne parlent pas ou peu aux adultes hors de la présence des enfants. On peut donc penser que la plupart des communications sont à but « éducatif » et que peu de place est consacrée à la concertation, la régulation professionnelle entre adultes.

Par ailleurs, si la fréquence des échanges avec la plupart des acteurs ne diffère guère en fonction du poste du matin et de l'après-midi, une forte variation apparaît dans le nombre d'interactions avec les enfants (Moy. matin 110 fois et Moy. après-midi 45 fois) et avec les enseignants (Moy. matin 15 fois et Moy. après-midi 3,6 fois). Ce qui correspond à la différence des missions et des activités entre ces deux parties de la journée (cf.§ 5.1).

Enfin, quelques échanges écrits ont été observés ${ }^{11}$ sous la forme de messages affichés sur les tableaux des classes ou de la salle de repos des ASEM. Ils s'apparentaient à des ordres formulés par les enseignants : «baisser les stores », «faire des crêpes, classe de Mme X», renvoyant semble-t-il les ASEM à leurs missions d'entretien et de restauration.

\subsection{3.- Une autonomie toute relative en fonction des missions}

L'autonomie, évaluée à partir d'items du questionnaire, est ici considérée au sens courant du terme, comme une possibilité d'organiser au quotidien son travail, moyennant le respect des règles générales du métier. Elle est considérée comme une condition favorisant le bien-être et la satisfaction au travail.

L'autonomie perçue dans le travail différencie également les missions puisque $51 \%$ des ASEM

11. relativement rares sur l'ensemble des observations ouvertes et systématiques. 
pensent ne pas pouvoir choisir la manière de travailler lors des phases d'assistance aux enseignants, alors que seulement $10 \%$ d'entre elles estiment ne pas être autonomes pour mener les missions de restauration et d'entretien. Cette autonomie est toutefois limitée par des règles d'hygiène et de sécurité qui encadrent les tâches, mais il n'y a personne pour diriger le travail au coup par coup. Ceci ne vaut pas pour la mission d'éducation où elles sont juridiquement considérées comme ne pouvant pas être responsables des enfants.

Un déséquilibre majeur du travail apparaît ici. La mission d'entretien, à laquelle les ASEM doivent consacrer le plus de temps est jugée la plus pénible, la moins intéressante malgré les possibilités relatives d'autonomie dans sa réalisation. La mission d'éducation, jugée la plus intéressante et peu pénible physiquement, laisse peu d'initiative dans son accomplissement, et est réduite en temps, alors même que la formation au CAP «petite enfance » valorise cette mission qui implique en principe des relations interpersonnelles (cf. § 2-1) qui semblent ici être pour le moins gênées sinon empêchées.

L'analyse conjointe des échanges verbaux et de l'autonomie perçue amène à conclure que :

- l'autonomie dans la réalisation des missions d'entretien et de restauration est réelle au quotidien, bien que contrainte par des règles strictement établies : les ASEM savent ce qu'elles ont à faire et comment; ce qui ne nécessite pas de concertation dans l'action;

- l'autonomie dans la réalisation de la mission éducative est très faible et témoigne d'une coupure, ou d'une mise à l'écart, de la « communauté éducative » dont les ASEM font officiellement partie.

\section{7.- Discussion - Conclusions}

Malgré un réel intérêt porté à la mission éducative, un sentiment partagé d'être soutenues par leur hiérarchie et suffisamment informées, les ASEM ne sont pas dans des conditions qui leur permettent de préserver leur santé et de construire un équilibre qui les satisfasse dans leur travail. Le cumul des sources de prescriptions du travail, les astreintes physiques et psychologiques, le manque de moyens matériels et humains, l'amoindrissement de la mission éducative (par ailleurs fortement investie par les ASEM) ou la mise à l'écart de la « communauté éducative » sont autant de dimensions de la pénibilité de ce travail. Elles ont ici des conséquences sur le bien-être au travail, la santé des ASEM et ceci de façon plus marquée chez les plus âgées.

Cette hypothèse forte de quasi confiscation du rôle éducatif est renforcée par les travaux de CauBareille $(2008$; 2009), menés auprès de professeurs des écoles de maternelle. À partir des entretiens, l'auteur fait plusieurs constats qui soulignent que spontanément, les enseignants évoquent très rarement la présence des ASEM dans les classes et leurs coopérations. Lorsqu'ils en parlent, ils reconnaissent leur statut de tiers dans la relation enseignant-élèves et le considèrent comme essentiel dans la régulation des tensions qui peuvent éclater dans la classe. Les enseignants regrettent cependant la présence souvent jugée éphémère des ASEM dans leur classe du fait d'une affectation à plusieurs classes en parallèle, ce qui « limite les processus de coopération» $(2008$, p. 14) à des aides auprès des enfants (habillage, passage aux toilettes, collation) plutôt qu'aux enseignants (préparation et animation d'ateliers en classe). Ils constatent, enfin, que les ASEM ont des niveaux de qualification très variés, rendant les possibilités de coopération aléatoires: «difficile à organiser, à optimiser » (p. 15).

Ces faits montrent le rapport ambivalent que peuvent entretenir les enseignants avec les ASEM, qu'ils considèrent parfois comme des femmes de ménage et non comme des partenaires du travail éducatif. Ils mettent aussi en évidence que l'appartenance à des institutions différentes d'une part et les organisations du travail proposées aux ASEM d'autre part, amènent ces deux catégories de professionnels de l'enfance à se croiser plus qu'à se voir et coopérer, à être disjointes plus que réunies dans une même communauté.

L'analyse fine du travail des ASEM permet de rejoindre les analyses de Kumar et Kumar (2008) 
ou de Pehkonen et al (2009) sur les pénibilités physiques des tâches d'entretien et de restauration. Elle alimente la description des contraintes organisationnelles auxquelles sont soumises les ASEM (Barbier-Le Déroff, 1998), précise les relations entre conditions de travail et bien-être au travail soulevées par le Floc'h (2006) ou Genest (2009) et pointe les difficultés grandissantes ressenties avec l'avancée en âge. Enfin, elle converge avec les travaux de Gorz (1988) et de Messing, Haentjens et Doniol-Show (1992) sur ces activités de service qui peuvent être assimilées à un travail de serviteur, se déroulant en l'absence du bénéficiaire dans bien des cas, et donc en toute transparence pour ce dernier.

Le cumul des missions, dont doivent s'acquitter les ASEM, peut être assimilé à une réelle polyvalence en termes de diversité des compétences nécessaires pour accomplir les tâches qui leur incombent. On est loin ici des travers d'une polyvalence basée sur un système de rotation sur différents postes de travail, dont les exigences physiques et cognitives sont similaires, qui n'a pour seul effet que de rompre la monotonie d'un travail souvent répétitif sans alléger les contraintes ni développer les compétences (Coutarel, Daniellou, \& Dugué, 2003). Toutefois, la polyvalence introduite dans le travail des ASEM limite les marges de manœuvre dont elles disposent en situation. En effet: comment faire vivre cette polyvalence a) lorsque le travail en sous-effectif est récurrent, mettant à mal le travail collectif des équipes de chaque école; b) lorsque les plus âgées n'ont pas initialement été recrutées et n'ont pour la plupart pas été formées pour assurer la mission éducative; c) alors que les ASEM sont mises à l'écart d'une « communauté éducative » qui pourrait être une ressource dans la mise en place de cette mission? Autant de points qui rejoignent et alimentent les constats faits dans le secteur industriel sur d'une part, les difficultés rencontrées et précautions nécessaires dans la mise en œuvre d'une polyvalence qui se soucie conjointement de l'efficacité du travail et de la préservation de la santé d'une part (Vézina, 2003) et sur, d'autre part, les particularités de la relation entre « avancée en âge et polyvalence » qui suppose des dispositifs de formation adaptés (Chatigny, 2001; Gaudart, 2003). Dans ce contexte, les conditions matérielles et organisationnelles de réalisation des trois missions provoquent ici un cumul d'astreintes qui induit une gestion plus ou moins difficile au quotidien de tâches interférentes, isole les ASEM et augmente les pénibilités du travail. En particulier, on peut faire l'hypothèse qu'un problème important du point de vue psychologique réside dans la contradiction entre l'autonomie dont les ASEM bénéficient justement dans les activités qu'elles jugent le moins valorisantes (restauration et entretien) par rapport aux gênes qu'elles ressentent dans leur fonction d'aide éducative que toutes valorisent.

La diversité des autorités qui prescrivent le travail des ASEM, associée au cumul des missions, les expose à des attentes et exigences très variées, potentiellement contradictoires et parfois difficiles à concilier. Par ailleurs, nous sommes ici face à une situation de relation de service qui nous semble originale et qui pourrait faire l'objet de nouvelles réflexions, par rapport à celles déjà menées sur la question (Cerf \& Falzon, 2005) : certains bénéficiaires (les enseignants ou, dans une moindre mesure, les animateurs) sont aussi des prescripteurs du travail des ASEM. Au-delà des prescriptions qu'ils font sur la mise en œuvre de la mission éducative auprès des enfants, puisque les ASEM ne sont pas directement responsables des enfants, il leur arrive de définir d'autres règles pour la mission d'entretien dont ils bénéficient. Par exemple les enseignants, que les ASEM assistent dans les classes ou pour lesquels elles font le ménage, prescrivent leur travail en définissant les règles de fonctionnement dans les classes, mais peuvent aussi intervenir sur les procédures de nettoyage. Les ASEM peuvent se retrouver à devoir gérer des instructions contradictoires; dont on sait qu'elles compliquent l'activité et peuvent constituer un facteur de mal-être ou de stress (Clot, 2008; Loriol, 2010). Ainsi, dans une école où les toilettes jouxtent le dortoir des enfants, la directrice exige de laver les sols, les éviers et les cuvettes sans tirer les chasses d'eau pour ne pas nuire au confort des enfants durant la sieste. Cette demande va à l'encontre des règles d'hygiène, garanties par la mairie, qui supposent que les eaux usées ne stagnent pas, a fortiori dans un lieu nettoyé. Elle met en porte à faux les ASEM qui n'ont pas le sentiment de faire, dans ces conditions, un travail de qualité. Une piste de recherche serait alors 
d'identifier quelles conséquences sur le bien-être et la santé au travail a le fait de travailler pour des bénéficiaires aussi prescripteurs du travail qu'elles sont amenées à faire (non au sens de la «prescription ascendante » proposée par Daniellou (2002) mais bien de «prescription descendante »).

Au-delà d'une description fine des pénibilités auxquelles sont soumises les ASEM de cette commune, l'étude a permis de remettre ensemble au travail des partenaires sociaux (élus, représentants syndicaux, responsables du service sociaux éducatif) qui campaient chacun sur leur position et de légitimer des plaintes des ASEM en apportant des éléments de compréhension de la situation vécue par celles-ci. Le clivage entre Fonction Publique Territoriale et Education Nationale, assumé par la mairie dans son refus d'associer un représentant des enseignants au comité de pilotage de l'étude, a à cette occasion été discuté, faisant bouger les représentations vers une vision plus intégrée du travail des ASEM avec celui des enseignants d'une part, et des animateurs d'autre part. Au sein du comité de pilotage, chargé dans la phase suivante du déploiement d'actions concrètes, il a été acté que ce travail constituait un socle de connaissances partagées sur les conditions de travail et de l'état de santé des ASEM à partir duquel il convenait d'agir tant sur les aspects matériels (mobilier, vêtements professionnels, etc.) que sur des aspects organisationnels permettant de créer les conditions de la mise en œuvre d'une polyvalence productive et protectrice, et de la construction d'une réelle «communauté éducative ». Les scénarios démographiques de vieillissement de la population ${ }^{12}$ (Molinié et Volkoff, 2002) sont venus rappeler que les questions des pénibilités au travail et d'aménagement du travail auprès d'une population âgée qui vieillira encore dans les années à venir (environ $36 \%$ d'ASEM âgées de plus de 50 ans) se posent donc de manière aiguë (Delgoulet, Mardon, Sinnassamy, $\&$ Weill-Fassina, 2009). Ce problème est d'autant plus important qu'il rencontre celui de l'évolution des carrières pour lequel il semble que les plus âgées aient peu de solutions alors que les plus jeunes envisagent des départs vers les postes d'animatrices ou d'assistantes de puériculture où elles pourront mieux valoriser leur CAP petite enfance (Delgoulet, Weill-Fassina, \& Mardon, 2010).

\section{REMERCIEMENTS}

Nous tenons à remercier Christelle Sinnassamy qui a réalisé le recueil des données à la mairie de V. dans le cadre de son Master recherche en Ergonomie. Nous remercions aussi les personnels, les responsables des services socio-éducatifs et des ressources humaines, les élus, et représentants syndicaux de cette mairie avec lesquels nous avons pu mener une recherche-action constructive.

\section{RÉFÉRENCES}

Arnaudo, B., Hamon-Cholet, S., \& Waltisperger, D. (2006). Contraintes posturales et articulaires au travail. Premières Informations et Synthèses, 11 (2).

Barbier-Le Déroff, M.A. (1998). Temporalités multiples et activité de travail: la situation des ATSEM. Actes du XXXIIIè congrès de la SELF (pp. 456-465), Paris.

Barbier-Le Déroff, M.A. (2007). Des grands dans l'espace des petits. Les agents spécialisés des écoles maternelles. Ethnologie française, 37(4), 655-662.

Cau-Bareille, D. (2007). Collectifs et âges. Rapport de recherche du CÉE No 37, 99-132

Cau-Bareille, D. (2008). Les fins de carrière en maternelle. Rapport interne du CREAPT-Centre d'Etudes et de l'Emploi, 65p.

Cau-Bareille, D. (2009). Vécu du travail et santé des enseignants en fin de carrière: une approche ergonomique. Rapport du CÉE n56, 68 p. http://www.cee-recherche.fr/fr/rapports/56-vecu-travail-sante-enseignants-carriere-ergonomique.pdf

12. À 2 ans et à 5 ans d'intervalles, effectif constant et âge de départ en retraite équivalent aux pratiques en vigueur dans cette commune au moment de l'étude (62 ans pour les plus âgées). 
Cerf, M., \& Falzon, P. (Eds.) (2005). Situations de service : travailler dans l'interaction. Paris: PUF.

Chatigny, C. (2001). Les ressources de l'environnement: au cœur de la construction des savoirs professionnels en situation de travail et de la protection de la santé. PISTES, 3(2). http://pettnt/pistes/ $\mathrm{v} 3 \mathrm{n} 2 / \mathrm{articles} / \mathrm{v} 3 \mathrm{n} 2 \mathrm{a} 5 \mathrm{htm}$

Clot, Y. (2008). Travail et pouvoir d'agir, Paris: PUF.

Coutarel, F., Daniellou, F. \& Dugué, B. (2003). Interroger l'organisation du travail au regard des marges de manœuvre en conception et en fonctionnement. La rotation est-elle une solution aux TMS ? PISTES, 5(2), http ://pettnt/pistes/v5n2/articles/v5n2a2.htm

Daniellou, F. (2002). Le travail des prescriptions. Actes du XXXVIIème congrès de la SELF. Les évolutions de la prescription, Aix-en Provence, pp. 8-15.

Daniellou, F. (2010). Les mondes du travail. In L. Théry (Ed), Le travail intenable (pp. 1-82), Paris: La Découverte (2 ${ }^{\text {ème }}$ édition).

De La Garza, C., \& Weill-Fassina, A. (2000). Régulations horizontales et verticales du risque. In T.H. Benchekroun, \& A.Weill-Fassina (Eds.), Le travail collectif: perspectives actuelles en ergonomie (pp. 217-234), Toulouse: Octarès Éditions.

Delgoulet, C., Mardon, C., Sinnassamy, C., \& Weill-Fassina, A. (2009). Pénibilités des activités et santé perçue des Agents Spécialisés des Ecoles Maternelles (ASEM). Des évolutions avec l'âge.

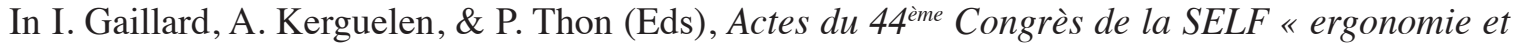
organisation du travail » (pp. 327-334), Toulouse, 22-24 septembre.

Delgoulet, C., Weill-Fassina, A., \& Mardon, C. (2010). Gérer les pénibilités de l'activité d'Agents Spécialisés des Ecoles Maternelles après 40 ans. Archives des maladies professionnelles et de l'environnement, 71(3), 363.

Falzon, P., \& Lapeyrière, S. (1998). L'usager et l'opérateur: ergonomie et relations de service. Le Travail Humain, 61(1), 69-90.

Gaudart, C. (2003). La baisse de la polyvalence avec l'âge : question de vieillissement, d'expérience, de génération? PISTES, 5(2). http :// http ://pettnt/pistes/v5n2/articles/v5n2a4.htm

Genest, B. (2009). Des agents de nettoyage des écoles confrontés à la didactique professionnelle. Travail et Apprentissage, 4, 57-74.

Gorz, A. (1988). Métamorphoses du travail. Critique de la raison économique. Paris: Gallimmard, folio essais. 439p.

Guérin, F., Laville, A., Daniellou, F., Duraffourg, J., \& Kerguelen, A. (1997). Comprendre le travail pour le transformer. La pratique de l'ergonomie. Lyon: Anact éditions.

Guilbert, M. (1966). Les fonctions des femmes dans l'industrie. La Haye: Mouton.

Guignon, N., \& Hamon-Cholet, S. (2003). Au contact avec le public, des conditions de travail particulières. Premières synthèse Insee, 9(3).

Ha, C., \& Roquelaure, Y. (2007). Réseau expérimental de surveillance épidémiologique des troubles musculo-squelettiques dans les Pays de Loire. Protocole de la surveillance dans les entreprises (2002-2004). Rapport de recherche, Mai, 87p.

Haukka, E., Leino-Arjas, P., Solovieva, S., Ranta, R., Viikari-Juntura, E., \& Riihimaki, H. (2006). Co-occurrence of musculoskeletal pain among female kitchen workers. International Archives of Occupational Environmenl and Health, 80, 141-148.

Ilmarinen, J. (1994). The ageing worker. Scandinavian Journal of Working Environment and Health, 18 (Suppl 2), 1-141.

Kerguelen, A. (2008). “Actogram Kronos”: un outil d'aide à l'analyse de l'activité. In H. Norimatsu, 
\& N. Pigem (Eds), Les techniques d'observation en sciences humaines (pp. 142-158). Paris: Armand Colin.

Kumar, H., \& Kumar, S. (2008). Musculoskeletal risk factors in cleaning occupation-A literature review. International Journal of Industrial Ergonomics, 38, 158-170.

Lasfargues, G. (2005). Départs en retraite et «travaux pénibles ». L’usage des connaissances scientifiques sur le travail et ses risques à long terme pour la santé. Rapport de recherche CÉE, $\mathrm{n}^{\circ} 19$, Avril.

Laursen, B., Sogaard, K., Sjogaard, G.(2003). Biomedical model predicting electromyographic activity in three soulder muscles from 3D kinematics and external forces during cleaning work. Clinical Biomecanichs, 18(4), 287-295.

Le Floc'h, V. (2006). Santé, travail, un couple sous surveillance pour les personnels éducatifs. In G. Fotinos (Ed), Le Climat des écoles primaires (pp. 208-219). Rapport de recherche pour la MGEN.

Le Robert (1989). Dictionnaire de la langue française.

Loriol, M. (2010) Agir contre le stress et les risques psychosociaux au travail. Regards sur l'actualité. $363,52-63$.

Messing, K., Chatigny, C., \& Courville, J. (1998). « Light » and « Heavy » work in the housekeeping service of a hospital. Applied Ergonomics, 29(6), 451-459.

Messing, K., Haentjens, C., \& Doniol-Shaw, G. (1992). L'invisible nécessaire: l'activité de nettoyage des toilettes sur les trains de voyageurs en gare. Le Travail Humain, 55, 353-370.

Molinié A.F. (2005). Enquête «Santé et Vie professionnelle après 50 ans». Résultats par secteur d'activité. Rapport pour le Conseil d'orientation des retraites. Rapport de recherche du Centre d'études de l'emploi, n²6, Octobre.

Molinié, A.F., \& Volkoff, S. (2002). La démographie du travail pour anticiper le vieillissement. Lyon: Editions ANACT, collection « Outils et méthodes ».

Molinié, A.F., \& Volkoff, S. (2003). Départs en retraite : les deux facettes de la «pénibilité » au travail. Quatre pages $d u C E E, \mathrm{n}^{\circ} 60$, novembre.

Molinié, A.F., \& Volkoff, S. (2006). Fin de vie active et pénibilités du travail. In Y. L'Horty (Ed.), la qualité de l'emploi (pp. 95-104). Paris: La Découverte.

Pehkonen, I., Takala, E.-P., Ketola, R., Viikari-Juntura, E., Leino-Arjas, P., Hopsu, L., Virtanen, T., Haukka, E., Holtari-Leino, M., Nykyri, E., \& Riihimäki, H. (2009). Evaluation of a participatory ergonomic intervention process in kitchen work. Applied Ergonomics, 40, 115-123.

Pekkarinen, A., \& Anttonen, H. (1988). The effect of working height on the loading of the muscular and skeletal systems in the kitchens of workplace canteens. Applied Ergonomics, 19(4), 306-308.

Petit, H. (1994). L'agent spécialisé à l'école maternelle (ASEM). Paris: ESF.

Prunier-Poulmaire, S., \& Gadbois, C. (2005). Quand le questionnaire s'impose à l'ergonomie. In S. Volkoff (Ed.), L'ergonomie et les chiffres de la santé au travail : ressources, tensions et pièges (pp. 75-86). Toulouse: Octarès Editions.

Puech, I. (2007). Cleaning time, protest time: employment and working conditions for hotel maids, Sociologie du Travail, 49(Suppl 1), 50-65.

Rey, A., \& Rey-Debove, J. (Eds.) (1989). Le Petit Robert: dictionnaire alphabétique et analogique de la langue française. Paris: Dictionnaires Le Robert éditions.

Rogard, V. (1997) L'intervention. In,M. de Montmollin (Ed.), Vocabulaire de l'Ergonomie (pp. 175179). Toulouse: Octarès Editions.

Struillou, Y. (2003). Pénibilité et retraite. Rapport pour le Conseil d'Orientation des Retraites. Avril. 
Vézina, N. (2003). Implantation de la rotation: quels sont les enjeux? Quelles sont les balises? PISTES, 5(2). http :// http ://pettnt/pistes/v5n2/articles/v5n2a9.htm

Weller, M. (1999). L'Etat au guichet, sociologie cognitive du travail et modernisation administrative des services publics. Paris: Desclée de Brouwer.

Wisner, A. (1974). Contenu des tâches et charge de travail. Sociologie du travail, 4, 339-357.

Woods, V., \& Buckle, P. (2005). An investigation into the design and use of workplace cleaning equipment. International Journal of Industrial Ergonomics, 35(3), 247-266.

\begin{abstract}
RÉSUMÉ
L'objectif de cette étude, réalisée auprès d'une mairie, était de comprendre en quoi les conditions de travail d'Agents Spécialisés des Ecoles Maternelles (ASEM) qui ont officiellement des missions d'entretien des locaux, de restauration et d'éducation auprès des enfants, pouvaient être source des pénibilités dont elles se plaignaient. Le but à plus long terme était de réfléchir à des pistes d'action pour prévenir la survenue de maladies et accidents du travail et surtout d'anticiper les conséquences du vieillissement de cette population tant en termes d'amélioration des conditions de travail que de gestion du personnel. Les investigations s'appuient sur une diversité de travaux antérieurs concernant le concept de pénibilité(s), les spécificités d'activités professionnelles proches (entretien, de locaux, restauration collective) et les évolutions avec l'âge. L'hypothèse principale porte sur les effets liés au cumul et à la diversité des missions. Les méthodes d'analyse du travail utilisées sont complémentaires: entretiens exploratoires, observations et questionnaires de santé perçue. Elles permettent de repérer les sources de pénibilités du travail et leur évolution avec l'avancée en âge des agents. Les résultats concordent pour montrer que malgré un réel intérêt porté au travail par les ASEM, leurs différentes missions sont chacune source de difficultés; leur cumul et leur diversité y ajoutent des problèmes de tâches interférentes et de valeurs relatives de leurs activités. Si l'on considère l'activité collective au sein des écoles, il apparaît que la diversité des institutions et des prescripteurs dont elles dépendent ne favorise pas les coordinations et qu'elles sont isolées de la « communauté éducative » dont elles font partie formellement; ce qui renforce encore les pénibilités du travail. Ces différents problèmes semblent s'accentuer avec l'âge.
\end{abstract}

\title{
MotS-CLÉS
}

Organisation du travail, activités de service, pénibilités, santé, âge, Agent Spécialisé des Écoles Maternelles.

\section{RÉFÉRENCEMENT}

Delgoulet, C., Weill-Fassina, \& A., Mardon, C. (2011). Pénibilités des activités de service et santé des Agents Spécialisés des Écoles Maternelles. Des évolutions avec l'âge. Activités, 8(1), pp. 2-25, http://www. activites.org/v8n1/v8n1.pdf

Article soumis le 22 octobre 2010, accepté pour publication le 26 février 2011 